\title{
AVALIAÇÃO DO ASSOALHO PÉLVICO DE ATLETAS: EXISTE RELAÇÃO COM A INCONTINÊNCIA URINÁRIA?
}

\author{
EVALUATION OF ATHLETES' PELVIC FLOOR: IS THERE A RELATION WITH URI
EVALUACIÓN DEL PISO PÉLVICO DE ATLETAS: ¿HAY UNA RELACIÓN CON \\ LA INCONTINENCIA URINARIA?
}

Maíta Poli de Araujo' (Médica)

Tathiana R Parmigiano' (Médica)

Laura Grechi Della Negra'

(Fisioterapeuta)

Luiza Torelli' (Fisioterapeuta)

Camila Garcia de Carvalho

(Fisioterapeuta)

Liris Wo' (Fisioterapeuta)

Aline Cristina Arrifano Manito ${ }^{1}$

(Medica)

Manoel João Batista Castello Girão (Médico)

Marair Gracio Ferreira Sartori (Médico)

1. Universidade Federal de São Paulo, Escola Paulista de Medicina, Setor de Ginecologia do Esporte, São Paulo, SP, Brasil.

\section{Correspondência:}

Rua Loeefgren 1570, Vila Mariana, São Paulo, Brasil. 01403003.

dramaita@gmail.com

\section{RESUMO}

Introdução: O assoalho pélvico feminino é formado por um conjunto de músculos, fáscias e ligamentos que sustentam os órgãos pélvicos (bexiga, ânus e vagina). A lesão destas estruturas, devido à idade avançada e parto podem levar à incontinência urinária. Entretanto, ainda não se sabe se o exercício de alta intensidade é capaz de lesar este conjunto músculo-aponevrótico, levando à incontinência urinária. Objetivo: Comparar a capacidade de contração dos músculos do assoalho pélvico entre mulheres sedentárias e atletas de elite, e verificar se existe associação com a ocorrência de incontinência urinária durante a atividade física. Métodos: Estudo caso-controle que incluiu 93 mulheres (49 atletas e 44 sedentárias) convidadas a responder o questionário "International Consultation on Incontinence Questionnaire - Short Form" e realizar avaliação funcional do assoalho pélvico. As atletas (grupo caso) eram praticantes de corrida de longa distância, basquete e ginástica olímpica. As sedentárias (grupo controle) realizavam menos de 150 minutos por semana de atividades moderadas ou vigorosas. A aferição da pressão de contração foi feita por meio de um perineômetro digital de precisão. Resultados: Os grupos foram homogêneos quanto à idade e índice de massa corpórea. As atletas apresentaram maiores valores de pressão vaginal máxima $\left(70,1 \pm 2,4 \mathrm{cmH}_{2} \mathrm{O}\right)$ quando comparadas às sedentárias $\left(34,3 \pm 1,7 \mathrm{cmH}_{2} \mathrm{O}\right),(\mathrm{p}<0,001)$. As atletas praticantes de basquete tiveram os maiores valores da pressão vaginal máxima $\left(77,2 \mathrm{cmH}_{2} \mathrm{O}\right)$ quando comparadas às ginastas $\left(65,5 \mathrm{cmH}_{2} \mathrm{O}\right)$ e corredoras $\left(65,4 \mathrm{cmH}_{2} \mathrm{O}\right)$. A prevalência de incontinência urinária nas atletas foi de $76 \%$ e somente $16 \%$ nas sedentárias $(p=0,005)$. Conclusão: Embora a capacidade de contração do assoalho pélvico em atletas de elite seja superior às sedentárias, a prevalência de incontinência urinaria foi elevada neste grupo de praticantes de esporte de alto rendimento e alto impacto.

Palavras-chave: incontinência urinária, esportes, diafragma da pelve.

\section{ABSTRACT}

Introduction: The female pelvic floor is formed by a set of muscles, fasciae, and ligaments that sustain pelvic organs (bladder, anus and vagina). Dysfunction of these structures, due to aging and childbirth may lead to urinary incontinence. However, we still do not know if high intensity exercises can damage this musculoaponevrotic set, leading to urinary incontinence. Objective: To compare the ability of contraction of pelvic floor muscles among sedentary women and elite athletes, and verify if there is an association with the occurrence of urinary incontinence during physical activity. Methods: Case-control study that included 93 women (49 athletes and 44 sedentary) invited to respond to the questionnaire "International Consultation on Incontinence Questionnaire - Short Form" and carry out functional evaluation of the pelvic floor. The athletes (case group) were practitioners of long distance running, basketball and Olympic gymnastics. Sedentary women (control group) performed less than 150 minutes of moderate or vigorous activities weekly. Contraction pressure was measured by a digital precision perineometer. Results: The groups were homogeneous as to age and body mass index. The athletes showed higher maximal vaginal pressure values $\left(70.1 \pm 2.4 \mathrm{cmH}_{2} \mathrm{O}\right)$ compared to sedentary women $\left(34.3 \pm 1.7 \mathrm{cmH}_{2} \mathrm{O}\right),(p<0.001)$. Athletes practitioners of basketball had the highest values of maximum vaginal pressure $\left(77.2 \mathrm{cmH}_{2} \mathrm{O}\right)$ compared to gymnasts $\left(65.5 \mathrm{cmH}_{2} \mathrm{O}\right)$ and runners $\left(65.4 \mathrm{cmH}_{2} \mathrm{O}\right)$. The prevalence of urinary incontinence in female athletes was of $76 \%$ and only $16 \%$ in sedentary women $(p=0.005)$. Conclusion: Although the ability to contract the pelvic floor in elite athletes is higher when compared with sedentary women, the prevalence of urinary incontinence is high in this group of practitioners of high performance and high impact sport.

Keywords: urinary incontinence, sports, pelvic floor.

\section{RESUMEN}

Introducción: El piso pélvico de las mujeres éformado por un conjunto de músculos, fascias y ligamentos que sostienen los órganos pélvicos (vejiga, el ano y la vagina). El daño a estas estructuras, debido a la edad avanzada y el parto puede llevar a la incontinencia urinaria. Sin embargo, no se sabe si el ejercicio de alta intensidad puede dañar este conjunto músculo-apronevrótico, lo que lleva a la incontinencia urinaria. Objetivo: Comparar la capacidad de contracción de 
los músculos del piso pélvico entre mujeres sedentarias y atletas de elite e investigar la asociación con la aparición de la incontinencia urinaria durante la actividad física. Métodos: Estudio de caso-controle que incluyó a 93 mujeres (49 atletas y 44 sedentarias) invitadas a responder al cuestionario "International Consultation on Incontinence Questionnaire - Short Form" y realizar la evaluación funcional del piso pélvico. Las atletas (grupo caso) eran practicantes de carrera de larga distancia y gimnasia olímpica. Las sedentarias (grupo control) realizaban menos de 150 minutos por semana de actividades moderadas o vigorosas. La medición de la presión de contracción fue por medio de un perineómetro digital de precisión. Resultados: Los grupos fueron homogéneos en el índice de masa corporal y la edad. Las atletas presentaran mayor presión máxima vaginal $\left(70,1 \pm 2,4 \mathrm{~cm} \mathrm{H}_{2} \mathrm{O}\right)$ en comparación con las sedentarias $\left(34,3 \pm 1,7 \mathrm{~cm} \mathrm{H}_{2} \mathrm{O}\right),(p<0,001)$. Las atletas practicantes de baloncesto tuvieron los valores más altos de presión vaginal máxima $\left(77,2 \mathrm{~cm} \mathrm{H}_{2} \mathrm{O}\right)$ en comparación con las gimnastas $\left(65,5 \mathrm{~cm} \mathrm{H}_{2} \mathrm{O}\right)$ y las corredoras $\left(65,4 \mathrm{~cm} \mathrm{H}_{2} \mathrm{O}\right)$. La prevalencia de la incontinencia urinaria en las atletas fue del $76 \%$ y sólo el $16 \%$ en las sedentarias $(p=0,005)$. Conclusión: Aunque la capacidad de contracción del piso pélvico en atletas de élite sea más alto que en sedentarias, la prevalencia de la incontinencia urinaria fue mayor en este grupo de practicantes de deporte de alto rendimiento e alto impacto.

Palabras clave: incontinencia urinaria, deportes, diafragma pélvico.

\section{INTRODUÇÃO}

Em mulheres, o esporte de alto rendimento e alto impacto aumenta em nove vezes o risco para incontinência urinária ${ }^{1,2}$. O risco para incontinência urinária (IU) em atletas depende muito da modalidade esportiva ${ }^{3-5}$. Assim, o risco é elevado em praticantes de corrida de longa distância, basquete e ginástica olímpica e é baixo no golfe, remo e ciclismo (quadro 1).

O impacto causado pela incontinência abrange a esfera sexual, social, doméstica e ocupacional. Mulheres incontinentes sentem-se constrangidas para a realização de atividades sociais e esportivas, e menos atraídas para o relacionamento sexual. Estudos mostram que estas pacientes apresentam sintomas depressivos, acompanhados de diminuição da autoestima e aumento da ansiedade ${ }^{6}$.

Existe pouco conhecimento acerca do funcionamento dos músculos do assoalho pélvico durante a prática desportiva. Na maioria dos exercícios em que ocorre aumento da pressão intra-abdominal, não existe contração voluntária desses músculos, motivo que explicaria a perda involuntária de urina ${ }^{7}$. Por outro lado, injúrias diretas ao assoalho pélvico, associado ou não a alterações hormonais e alimentares, poderiam comprometer a capacidade de contração destes músculos, predispondo a IU $U^{1,7}$.

Assim, a avaliação clinica e funcional do assoalho pélvico em atletas é fundamental para a prevenção ou tratamento da IU ${ }^{8}$. Entende-se como avaliação funcional a visualização e medida da contração dos músculos do assoalho pélvico (MAP), sobretudo o músculo levantador do ânus ${ }^{9}$. A visualização da contração pode ser realizada por simples observação durante o exame ginecológico ou por meio de dispositivos: ultrassom, ressonância magnética ou eletromiografia ${ }^{10}$. Já a medida da contração pode ser feita pelo toque vaginal (manobra digital) ou com perineômetros, dinamômetros ou cones vaginais ${ }^{11}$.

A manobra digital é fácil de aprender, barata e causa pouco incômodo à atleta ${ }^{11}$. Consiste de um toque vaginal, com a introdução dos dedos indicador e médio, com os músculos relaxados. Solicita-se a atleta uma contração muscular máxima ao redor do dedo do examinador e realiza-se uma gradação subjetiva com uma escala que normalmente varia de zero (pior contração) a cinco (contração máxima).

Quadro 1. Modalidades esportivas e risco para incontinência urinária.

\begin{tabular}{|c|c|c|}
\hline Alto risco & Risco moderado & Baixo risco \\
\hline Corrida & Tênis & Golfe \\
\hline Ginástica & Esqui & Remo \\
\hline Basquetebol & Voleibol & Ciclismo \\
\hline
\end{tabular}

Os perineômetros são dispositivos mecânicos, introduzidos na vagina, e avaliam de forma objetiva a função do musculo levantador do ânus. A gradação da força é dada de forma indireta por meio da pressão exercida sobre o probe vaginal, além de ser possível verificar a duração da contração em segundos ${ }^{12}$.

O uso de escalas de avaliação funcional e de equipamentos padronizados de medida da contração do assoalho pélvico permite a comparação de resultados em diferentes populações. Em atletas, esta avaliação é importante para a prevenção da incontinência urinaria.

Tendo em vista o impacto negativo da IU na qualidade de vida e desempenho de mulheres atletas, e da importância da avaliação do assoalho pélvico em esportes de alto rendimento e alto impacto, este trabalho teve como objetivo avaliar a capacidade de contração do assoalho pélvico, a prevalência de incontinência urinaria em atletas de elite e se existe associação entre a forca muscular e a ocorrência da incontinência urinaria.

\section{MÉTODOS}

Estudo caso-controle realizado no Setor de Ginecologia do Esporte da Escola Paulista de Medicina, Universidade Federal de São Paulo (EPM-UNIFESP) e aprovado pelo Comitê de Ética em Pesquisa da mesma Instituição sob o número 1709/10..

Participaram da pesquisa 93 mulheres, no menacme, nulíparas, eumenorreicas e que assinaram o Termo de Consentimento Livre e Esclarecido (TCLE).

O grupo caso foi formado por 49 atletas praticantes de esporte de alto rendimento e alto impacto. Neste grupo foram incluídas corredoras de longa distância, jogadoras de basquete e ginastas. Todas as participantes eram Federadas e tinham experiência há no mínimo cinco anos.

O grupo controle foi constituído por 44 sedentárias, de acordo com - Questionário Internacional de Atividade Física (International Physcal Activity Questionary - $(P A Q)^{13}$. Para que estas mulheres fossem incluídas neste grupo, elas deviam realizar menos de 150 minutos por semana de atividade física ${ }^{14}$.

Os grupos foram homogêneos quanto à idade, circunferência abdominal, índice de massa corpórea (IMC) e cor da pele (autoreferidas) (tabela 1).

A avaliação clinica foi feita pela análise da queixa de incontinência urinaria nas atletas. Assim, utilizou-se o questionário "International Consultation of Incontinence Questionnaire - Short Form (ICIQ-SF)", traduzido e validado para a língua portuguesa ${ }^{15,16}$. O ICIQ-SF é um questionário simples, composto por questões que avaliam a frequência, 
gravidade e o impacto da incontinência urinária. O resultado do ICIQ-SF igual a zero determina ausência de incontinência urinária ${ }^{17}$. O ICIQ-SF com escore maior ou igual a oito é considerado como a melhor nota de corte para diferenciar pacientes que se incomodam com a IU daqueles que não se incomodam (sensibilidade de $64 \%$ e especificidade de $91 \%)^{17}$.

A avaliação funcional dos MAP foi realizada pela manobra digital e perineometria. Procedeu-se ao toque vaginal, com a introdução dos dedos indicador e médio, com os músculos relaxados. Posteriormente, foi solicitado às participantes que contraíssem e mantivessem a contração dos músculos ao redor do dedo do examinador com o máximo de força que conseguissem. A gradação da capacidade de contração foi dada em graus de avaliação funcional (AFA), que vai de zero (AFA 0 ) até cinco (AFA 5) ${ }^{18}$. Esta classificação associa a visualização e a medida da contração dos MAP. A visualização da contração denomina-se função perineal objetiva, e a sensibilidade à palpação digital, denomina-se função perineal subjetiva.

A aferição da pressão de contração foi feita por meio de um perineômetro digital de precisão (marca Peritron $9300^{\circledR}$, fábrica Cardio-Design, Austrália). O equipamento é composto por um sensor de borracha siliconado de $26 \mathrm{~mm}$ de largura conectado a um tubo de plástico com $80 \mathrm{~cm}$ de comprimento, e uma unidade leitora (figura 1). O sensor é ativado de acordo com a contração da musculatura do assoalho pélvico e a pressão é transferida para a unidade leitora, onde o registro é dado em centímetros de água $\left(\mathrm{cm} \mathrm{H}_{2} \mathrm{O}\right)$. O equipamento é leve e funciona com pilha. A leitura do tem alto índice de acurácia, com um erro de medida de $\pm 3 \mathrm{~cm} \mathrm{H}_{2} \mathrm{O}$. Este pequeno erro é quase nulo quando a pressão ao repouso é igual a zero antes da solicitação da contração dos músculos do assoalho pélvico ${ }^{19}$.

Para introdução da sonda vaginal é necessário que a mesma seja protegida com um preservativo masculino não lubrificado, uma vez que não é possível esterilizá-la ${ }^{20}$. Utilizou-se gel lubrificante na parte externa do preservativo masculino a fim de facilitar a introdução da sonda. 0 sensor de pressão foi introduzido na vagina, e automaticamente foi

Tabela 1. Comparação das variáveis demograficas entre as atletas e sedentárias.

\begin{tabular}{c|c|c|c}
\hline Variável & Atleta $(\mathbf{N}=\mathbf{4 9})$ & Sedentária $(\mathbf{N}=\mathbf{4 4})$ & Valor de $\mathbf{p}$ \\
\hline Idade & $20 \pm 3$ & $21 \pm 4$ & 0,15 \\
\hline IMC & $21,3 \pm 2$ & $22 \pm 2,4$ & 0,08 \\
\hline Circunferencia abdominal & $71,6 \pm 4$ & $73,3 \pm 4$ & 0,3 \\
\hline Cor da pele (auto-referida) & & & \\
\hline Branca & 46 & 39 & 0,5 \\
\hline Preta ou parda & 3 & 5 & \\
\hline
\end{tabular}

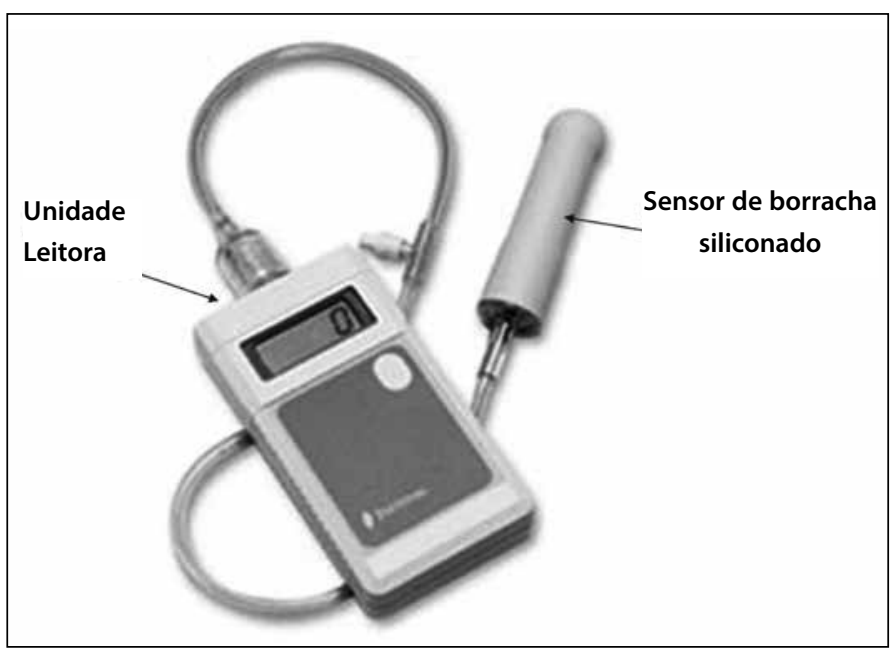

Figura 1. Perineometro digital da marca Peritron $9300^{\circ}$ quantificada a pressão vaginal de repouso (musculatura em repouso). O aparelho foi zerado e solicitou-se uma contração máxima, por pelo menos três segundos (pressão vaginal máxima)21.

A análise estatística foi realizada pelo software GraphPad Prism 4. A comparação entre dois grupos com variáveis quantitativas que apresentaram distribuição normal e variância semelhante foi realizada pelo teste $t$ de Student; caso contrário foi feito pelo teste de Mann-Whitney U. A comparação das variáveis qualitativas foi feita pelo Teste de Qui-Quadrado $\left(X^{2}\right)$. Os dados contínuos de mais de dois grupos foram comparados pela Análise de Variância (ANOVA) Considerou-se $p<0,05$ como nível de significância.

\section{RESULTADOS}

A avaliação funcional do assoalho pélvico por meio da palpação digital mostrou diferença estatisticamente significante entre o grupo de atletas e sedentárias (tabela 2). A maioria das atletas apresentou AFA superior a três e nenhuma sedentária apresentou AFA 5.

$O$ resultado da perineometria pode ser observado na figura 2. Não houve diferença entre os grupos consoante a pressão vaginal de repouso. Contudo, as atletas apresentaram maiores valores de pressão vaginal máxima $\left(70,1 \pm 2,4 \mathrm{cmH}_{2} \mathrm{O}\right)$ quando comparadas as sedentárias $(34,3 \pm 1,7$ $\left.\mathrm{cmH}_{2} \mathrm{O}\right)$ e este resultado foi estatisticamente significante $(\mathrm{p}<0,001)$.

As atletas praticantes de basquete tiveram os maiores valores da pressão vaginal máxima $\left(77,2 \mathrm{cmH}_{2} \mathrm{O}\right)$ quando comparadas as ginastas $\left(65,5 \mathrm{cmH}_{2} \mathrm{O}\right)$ e corredoras $\left(65,4 \mathrm{cmH}_{2} \mathrm{O}\right)$ (figura 3). Contudo, estes valores não tiveram diferença estatisticamente significante $(p=0,05)$.

A prevalência de incontinência urinaria nas atletas foi de $76 \%$ e somente $16 \%$ nas sedentárias $(p=0,005)$. Contudo, mesmo as atletas incontinentes, tiveram valores elevados de pressão vaginal máxima quando comparado às sedentárias incontinentes (figura 4).

\section{DISCUSSÃO}

A prevalência de incontinência urinaria nas atletas deste estudo foi de $76 \%$, resultado muito superior ao encontrado em outras pesquisas com mulheres esportistas brasileiras.

Em 2008, o Setor de Ginecologia do Esporte da EPM-UNIFESP avaliou 37 corredoras de longa distância, com media de idade de 35 anos por meio do questionário ICIQ-SF. A prevalência da IU foi de 62,2\% e a perda urinaria ocorria tanto na competição como no treino ${ }^{7}$.

Antunes et al. ${ }^{22}$ entrevistaram 50 praticantes de academia, com faixa etária entre 25 e 50 anos, da cidade de Taubaté-SP. A idade média das

Tabela 2. Comparação entre as mulheres atletas e sedentarias consoante a pressão vaginal de repouso e a pressão vaginal máxima (valores em cmH2O).

\begin{tabular}{c|c|c|c}
\hline Variável & Atleta & Sedentária & Valor de $\mathbf{p}$ \\
\hline Pressão de repouso & $37,7 \pm 8$ & $37,4 \pm 7$ & 0,8 \\
\hline Pressão Máxima & $70,1 \pm 2,4$ & $34,3 \pm 1,7$ & $<0,001$ \\
\hline
\end{tabular}

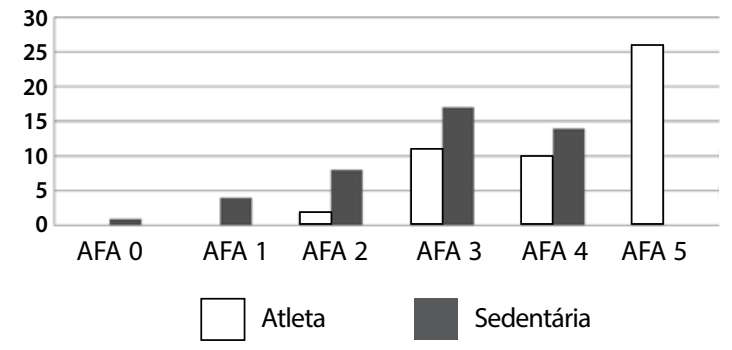

Figura 2. Comparação da gradação de avaliação funcional pela manobra digital entre atletas e sedendárias. 


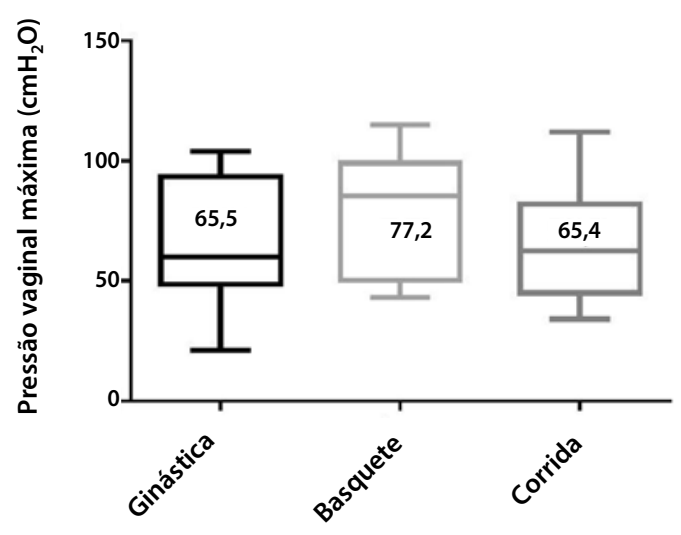

Figura 3. Comparação do valor da pressão vaginal máxima entre as modalidades esportivas.

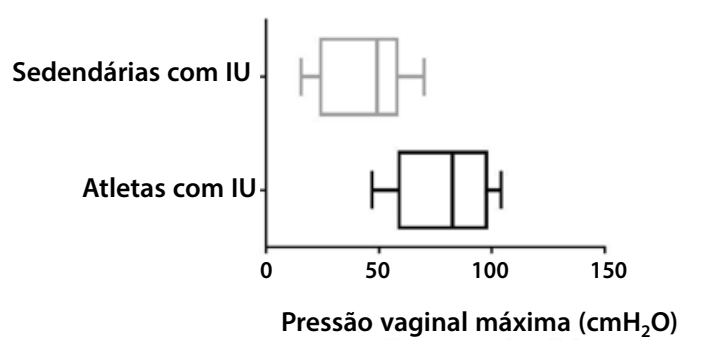

Figura 4. Comparação do valor da pressão vaginal máxima pela perineômetria entre atletas e sedentárias incontinentes.

participantes foi de 37 anos e a prevalência de IU foi de $36 \%$. O resultado do presente estudo foi alarmante, tendo em vista que as participantes eram mais jovens (média de 20 anos), com índice de massa corpóreo adequado e sem filhos.

A atividade física de alto impacto e alto rendimento é um fator importante de permanência de IU no futuro ${ }^{23}$. Estudo realizado com ex-atletas de trampolim acrobático mostrou que, as ginastas que tinham perda involuntária de urina quando jovens, 76\% continuaram a perder, mesmo após a interrupção do esporte. Os principais fatores de risco para a permanência de IU foram à inabilidade de interromper a urina $(p<0.001)$, constipação $(p=0.007)$, e inicio do treinamento antes da menarca $(p<0.001)^{24}$.

Consenso também existe acerca da forte correlação entre a modalidade desportiva e a incidência dos sinais e sintomas de perda urinária. A literatura aponta risco elevado nas praticantes de academia e musculação, de maneira isolada ou combinada ${ }^{7,25}$. Ademais, quase $80 \%$ das jovens atletas de trampolim acrobático (media de 15 anos) relatam perda involuntária de urina, mesmo durante um treino curto (15 minutos) ${ }^{6}$.

Os principais fatores de risco para o desenvolvimento de incontinência urinária feminina são: idade, gestação, paridade, cirurgias ginecológicas e constipação intestinal ${ }^{25}$. Estes eventos comprometem o assoalho pélvico, por enfraquecerem os músculos (levantador do ânus), as fáscias e os ligamentos. Contudo, estes fatores de risco não são encontrados em atletas incontinente, que normalmente são jovens e não tem filhos.

O resultado da avaliação funcional do assoalho pélvico mostrou que as atletas tem pressão vaginal máxima muito maior do que as sedentárias $(70,1 \pm 2,4$ vs. 34,3 $\pm 1,7, p<0,001)$. Do mesmo modo, a avaliação por meio da manobra digital evidenciou que as atletas tem função perineal subjetiva superior as sedentárias. A pressão vaginal traduz

de forma indireta a força do músculo levantador do ânus. Em atletas, imagens de ressonância magnética, evidenciam que a espessura deste músculo é muito maior do que mulheres sedentárias pareadas para idade e paridade ${ }^{26}$.

Comparando os valores da pressão vaginal máxima consoante à modalidade esportiva, embora as jogadoras de basquete tenham apresentado valores maiores $\left(77,2 \mathrm{CmH}_{2} \mathrm{O}\right)$ quando comparadas as corredoras $\left(65,4 \mathrm{cmH}_{2} \mathrm{O}\right)$ e ginastas $\left(65,5 \mathrm{cmH}_{2} \mathrm{O}\right)$, a diferença não foi estatisticamente significante. Resultado semelhante foi encontrado no estudo de Reis et al., que examinaram 20 atletas praticantes de voleibol e basquetebo ${ }^{27}$. A avaliação funcional destas atletas também foi feita pela gradação de AFA mas para a perineomteria utilizou-se um Biofeedback Eletromiográfico. Como resultado, a IU esteve presente em 50\% das jogadoras de basquete e em 30\% das jogadoras de vôlei e não houve diferença entre a capacidade de contração dos MAP tanto pela manobra digital como pelo biofeedback.

No presente estudo, embora a capacidade de contração do musculo levantador do ânus estivesse preservada, houve prevalência elevada de IU nas atletas. Assim, parece que outros fatores estão relacionados à perda de urina durante a prática desportiva.

Em ginastas e jogadoras de basquete, a principal explicação para a perda involuntária de urina relaciona-se com o deslocamento do assoalho pélvico durante os saltos e mudanças de direção ${ }^{28}$. Neste caso, seria necessário uma pré-contração muscular durante a atividade física, para neutralizar o deslocamento do assoalho pélvico e impedir a perda de urina.

Em esportes de endurance, como na corrida de longa distância, a IU talvez ocorra pela fadiga muscular. Isto porque cerca de $70 \%$ das fibras musculares do assoalho pélvico são do tipo I, ou seja, fibras de contração lenta, ricas em mitocôndrias, e que se contraem por mecanismo oxidativo ${ }^{29}$. Fatores que comprometem o suprimento de oxigênio para estas fibras promovem também diminuição da sua capacidade contrátil e fibras do tipo II são recrutadas. Estas, por serem fibras de contração rápida, não têm a mesma eficiência que as do tipo I para manter o tônus muscular do assoalho pélvico comprometendo o mecanismo de continência?.

Outras hipóteses que tentam explicar a fisiopatologia da IU em atletas envolvem os distúrbios hormonais, secundários a disponibilidade energética diminuída e/ou distúrbios menstruais. A amenorréia hipotalâmica, decorrente do exercício físico intenso, associada a desordens alimentares (anorexia ou bulimia) ou a combinação de ambos, levaria a baixos níveis de estrogênios, contribuindo para a IU em atletas?.

Tendo em vista a elevada prevalência de IU em atletas praticantes de esporte de alto impacto, e a provavel associação com o deslocamento do assoalho pélvico e não a fraqueza muscular, as atletas devem ser ensinadas a realizarem uma pré-contração ou uma contração simultânea dos músculos do assoalho pélvico durante a realização do exercício. Essas orientações são necessárias, uma vez que estudos verificaram que um terço das mulheres não consegue contrair estes músculos ${ }^{30}$.

Treinadores devem encorajar as atletas a realizarem a contração muscular durante suas atividades regulares, pois, sem orientação, é improvável que elas pensem a respeito dessa contração voluntariamente ${ }^{31}$.

A fisioterapia especifica para mulheres atletas é efetiva na prevenção e tratamento da IU com taxa de cura subjetiva ao redor de 70\% e taxa de cura objetiva de $67 \% \%^{30}$. Como as atletas estão acostumadas ao treinamento regular, adicionar protocolos de protecao ao assoalho pelvico, tres a quatro vezes por semana, não seria uma grande sobrecarga, permitindo contrações eficientes quando do aumento repentino da pressão intra-abdominal ${ }^{29}$. 
CONCLUSÃO

A prevalência de incontinência urinária em ginastas, jogadoras de basquete e corredoras de longa distância é elevada. Contudo, como a capacidade de contração do musculo levantador do ânus esta pre- servada, outros fatores devem estar envolvidos na etiopatogenia da incontinência urinaria em atletas.

Todos os autores declararam não haver qualquer potencial conflito de interesses referente a este artigo.

\section{REFERÊNCIAS}

1. Bø K, Borgen JS. Prevalence of stress and urge urinary incontinence in elite athletes and controls. Med Sci Sports Exerc. 2001;33(11):1797-802.

2. Vitton V, Baumstarck-Barrau K, Brardjanian S, Caballe I, Bouvier M, Grimaud JC. Impact of high-level sport practice on anal incontinence in a healthy young female population. J Womens Health (Larchmt). 2011;20(5):757-63.

3. Caetano AS, Tavares MCG, Lopes MHB. Incontinência urinária e a prática de atividades físicas. Rev Bras Med Esporte. 2007;13(4):270-4.

4. Thyssen $\mathrm{HH}$, Clevin L, Olesen S, Lose G. Urinary incontinence in elite female athletes and dancers. Int Urogynecol J Pelvic Floor Dysfunct. 2002;13(1):15-7.

5. Bø K. Urinary incontinence, pelvic floor dysfunction, exercise and sport. Sports Med. 2004;34(7):451-64.

6. Caetano AS, Tavares MCGCF, Lopes MHB, Poloni RL. Influência da atividade física na qualidade de vida e auto-imagem de mulheres incontinentes. Rev Bras Med Esporte. 2009;15(2):93-7.

7. Araujo MP, Oliveira E, Zucchi EVM, Trevisani VFM, Girão MBC, Sartori MGF. Relação entre incontinência urinária em mulheres atletas corredoras de longa distância e distúrbio alimentar. Rev Assoc Med Bras. 2008;54(2):146-9.

8. Rett MT, Simões JÁ, Herrmann V, Marques AA, Morais SS. Existe diferença na contratilidade da musculatura do assoalho pélvico feminino em diversas posições? Rev Bras Ginecol Obstet. 2005;27(1):20-3.

9. Bø K, Finckenhagen HB. Vaginal palpation of pelvic floor muscle strength: inter-test reproducibility and comparison between palpation and vaginal squeeze pressure. Acta Obstet Gynecol Scand. 2001;80(10):883-7.

10. Murad-Regadas SM, Bezerra LRP, Silveira CRS, Pereira JDR, Fernandes GOS, Vasconcelos Neto JA, et al. Características anatômicas e funcionais do assoalho pélvico em nulíparas avaliadas por ultrassonografia tridimensional endovaginal: estudo caso-controle e avaliação da confiabilidade interobservador. Rev Bras Ginecol Obstet. 2013;35(3):123-29.

11. Bø K, Sherburn M. Evaluation of female pelvic-floor muscle function and strength. Phys Ther. 2005;85(3):269-82

12. Isherwood PJ, Rane A. Comparative assessment of pelvic floor strength using a perineometer and digital examination. BJOG. 2000;107(8):1007-11.

13. Pardini R, Matsudo SMM, Araújo T, Matsudo VKR, Andrade E, Braggion G, et al. Validação do Questionário Internacional de Nível de Atividade Física (IPAQ - Versão 6): estudo piloto em adultos jovens brasileiros. Rev Bras Ciên e Mov. 2001;9(3):45-51.

14. Marcellino C, Henn RL, Olinto MT, Bressan AW, Paniz VM, Pattussi MP. Physical inactivity and associated factors among women from a municipality in southern Brazil. J Phys Act Health. 2014;11(4):777-83.

15. Tamanini JT, Dambros M, D'Ancona CA, Palma PC, Rodrigues Netto N Jr. Validation of the "International Consultation on Incontinence Questionnaire -- Short Form" (ICIQ-SF) for Portuguese. Rev Saude Publica. 2004;38(3):438-44.

16. Tamanini JT, Dambros M, D'Ancona CA, Palma PC, Rodrigues-Netto N Jr. Responsiveness to the Portu- guese version of the International Consultation on Incontinence Questionnaire-Short Form (ICIQ-SF) after stress urinary incontinence surgery. Int Braz J Urol. 2005;31(5):482-9.

17. Tamanini JTN, Tamanini MMM, Mauad LMQ, Auler AMB. Incontinência urinária: prevalência e fatores de risco em mulheres atendidas no Programa de Prevenção do Câncer Ginecológico. BEPA. 2006;3(34):17-23.

18. Contreras-Ortiz O, Coya Nuñez F, Gutnisky R, Cortese G. Perineal dysfunction dynamic assessment in women. Classification proposal. Obstet Gynecol Latin Amer. 1994;52:92-8.

19. Frawley HC, Galea MP, Phillips BA, Sherburn M, Bø K. Effect of test position on pelvic floor muscle assessment. Int Urogynecol J Pelvic Floor Dysfunct. 2006;17(4):365-71.

20. Da Roza T, Mascarenhas T, Araujo M, Trindade V, Jorge RN. Oxford Grading Scale vs manometer for assessment of pelvic floor strength in nulliparous sports students. Physiotherapy. 2013;99(3):207-11.

21. Barbosa PB, Franco MM, Souza Fde O, Antônio Fl, Montezuma T, Ferreira CH. Comparison between measurements obtained with three different perineometers. Clinics (Sao Paulo). 2009;64(6):527-33.

22. Antunes MB, Costa Manso VM, Andrade NVS. Análise dos sinais e sintomas da incontinência urinária de esforço em mulheres de 25 a 50 anos praticantes de atividades físicas em academias. Ensaios e Ciênc. 2011;15(1):83-95

23. Nygaard IE. Does prolonged high-impact activity contribute to later urinary incontinence? A retrospective cohort study of female Olympians. Obstet Gynecol. 1997;90(5):718-22.

24. Eliasson $\mathrm{K}$, Edner $\mathrm{A}$, Mattsson E. Urinary incontinence in very young and mostly nulliparous women with a history of regular organised high-impact trampoline training: occurrence and risk factors. Int Urogynecol J Pelvic Floor Dysfunct. 2008;19(5):687-96.

25. Marinho AR, Leal BB, Flister JS, Bernardes NO, Rett MT. Incontinência urinária feminina e fatores de risco. Rev Fisioter Bras. 2006;7(4):301-5.

26. Kruger JA, Murphy BA, Heap SW. Alterations in levator ani morphology in elite nulliparous athletes: a pilot study. Aust N Z J Obstet Gynaecol. 2005;45(1):42-7.

27. Reis AO, Camara CNS, dos Santos T, Dias TS. Estudo comparativo da capacidade de contração do assoalho pélvico em atletas de voleibol e basquetebol. Rev Bras Med Esporte. 2011;17(2):32-6.

28. Kruger JA, Dietz HP, Murphy BA. Pelvic floor function in elite nulliparous athletes. Ultrasound Obstet Gynecol. 2007;30(1):81-5.

29. Nygaard IE, Thompson FL, Svengalis SL, Albright JP. Urinary incontinence in elite nulliparous athletes Obstet Gynecol. 1994;84(2):183-7.

30. Hay-Smith EJ, Bø Berghmans LC, Hendriks HJ, de Bie RA, van Waalwijk van Doorn ES. Pelvic floor muscle training for urinary incontinence in women. Cochrane Database Syst Rev. 2001;(1):CD001407.

31. Da Roza T, de Araujo MP, Viana R, Viana S, Jorge RN, Bø K, et al. Pelvic floor muscle training to improve urinary incontinence in young, nulliparous sport students: a pilot study. Int Urogynecol J. 2012;23(8):1069-73 